\title{
Polarization-preserving angular shifter
}

\author{
Yi-Kai Cheng and Jyh-Long Chern \\ Department of Photonics, Institute of Electro-Optical Engineering \\ Microelectronics and Information System Research Center, \\ National Chiao Tung University, Hsinchu 300, Taiwan
}

\begin{abstract}
We propose two types of light pipes, namely wedge and circular forms, the incident direction of light propagation could be rotated as necessary while still preserving beam polarization. We also deduce the basic conditions of these preservations of polarization. Several typical cases of light pipes are analyzed by numerical simulation. As one of the examples, it is verified that a collimated beam with +45 degree linearly polarization can be guided to any pre-settled direction where the ellipse ratio variation in polarization is less than $0.1 \%$.
\end{abstract}

Keywords: Polarization, Light Pipe, Optical Design

\section{INTRODUCTION}

Preserving the polarization of a beam of light after propagation has many practical uses in a variety of applications such as biological tissue light probes or ellipsometers for refractive index and film thickness measurement, etc. [1,2]. When light is propagated, displaced, or deflected, its polarization is usually changed. For example, when light bounces off a reflective mirror, the reflection introduces an additional phase that alters the polarization of the light beam. Azzam suggested solutions to displace a monochromatic light beam parallel to itself without change of polarization by utilizing a pair of parallel mirrors with a single dielectric layer coating [3,4]. On the other hand, Cojocaru [5] and Wang et al. [6] proposed the design of coating layers with totally reflecting prism to provide a convenient and efficient method to control the phase retardance. Galvez presented the combinations of four total reflecting prisms to preserve polarization [7]. Galves' scheme has benefits in achromatic application and very good tolerance which can preserve the polarization with a square of ellipse ratio variation of less than $1 \%$ while one degree of component tilt occurs [8]. These studies provide very useful schemes to maintain polarization, but they generally focus on the displacement of a light beam, i.e., when the propagating direction of a displaced beam is parallel to the original beam. However, angular shifting is generally employed to reduce the optical path of beam propagation and hence the system size, which has practical value. Technically, polarization-preserving angular shifting provides more flexibility in optical engineering applications, e.g., polarized light probe with angular incident injection could provide another useful scheme in exploring 3D structure and response. However, it is quite uncertain and difficult to deflect the beam direction while maintaining the state of polarization using this technique. This study proposes and numerically verifies novel polarization-preserving beam angular shifters based on a wedge plate [9] and a circular-bent light pipe. A polarization-preserving wedge or bent light pipe has an advantage in manufacture and has better extendibility on the applications of polarization controlling

Nonimaging Optics and Efficient Illumination Systems $\mathrm{V}$,

edited by Roland Winston, R. John Koshel, Proc. of SPIE Vol. 7059

70590J, (2008) · 0277-786X/08/\$18 - doi: 10.1117/12.795848

Proc. of SPIE Vol. 7059 70590J-1 
mechanisms. A polarization-preserving wedge or bent light pipe can provide both spatial and angular shift of beam propagation path, and zero vertex angle/bent angle can reduce a wedge/light pipe angular shifter to become a beam displacer.

This paper is organized as follows. Section 2 provides basic formalism of polarized ray tracing in dielectric-filled material. Section 3 presents the issue of polarization conservation and its conditions. Section 4 shows the simulation verification. Section 5 gives the limitation of polarization-preserving angular shifter. Section 6 investigates the tolerance issue of manufacture accuracy and incident beam angle, and the final section contains conclusions.

\section{BASIC PROPAGATION FORMALISM OF POLARIZED LIGHT: THE MULLER MATRIX}

Let us first summarize the general properties of a polarized ray propagating in a dielectric-filled material. According to Fresnel's equations, the transmitted and reflected fields of a light beam on air-dielectric interface follows [10]

$$
\begin{gathered}
R_{s}=\frac{\cos \theta_{i}-n \cos \theta_{t}}{\cos \theta_{i}+n \cos \theta_{t}} E_{s}, R_{p}=\frac{n \cos \theta_{i}-\cos \theta_{t}}{n \cos \theta_{i}+\cos \theta_{t}} E_{p} \\
T_{s}=\frac{2 \cos \theta_{i}}{\cos \theta_{i}+n \cos \theta_{t}} E_{s}, T_{p}=\frac{2 \cos \theta_{i}}{n \cos \theta_{i}+\cos \theta_{t}} E_{p}
\end{gathered}
$$

In Eq. (1), $E, R$, and $T$ represent the incident, reflected, and refracted fields, and the subscripts s and $p$ denote the parallel and perpendicular directions, respectively. $\theta_{i}$ is the incident angle, $\theta_{t}$ is the angle of refraction, and $\mathrm{n}$ is the refractive index of the dielectric medium.

Stokes parameters make it possible to derive the Mueller matrix for the reflection and refraction on air-dielectric interface from Eq. (1). Referring to Ref. [11], the Mueller matrix of transmission on air-dielectric interface is

$$
M_{T}=\frac{\sin 2 \theta_{i} \sin \theta_{t}}{2\left(\sin \Theta_{+} \cos \Theta_{-}\right)^{2}}\left(\begin{array}{cccc}
\cos ^{2} \Theta_{-}+1 & \cos ^{2} \Theta_{-}-1 & 0 & 0 \\
\cos ^{2} \Theta_{-}-1 & \cos ^{2} \Theta_{-}+1 & 0 & 0 \\
0 & 0 & 2 \cos \Theta_{-} & 0 \\
0 & 0 & 0 & 2 \cos \Theta_{-}
\end{array}\right),
$$

where $\Theta_{ \pm}=\theta_{i} \pm \theta_{t}$, while for total internal reflection it follows that

$$
M_{T I R}=\left(\begin{array}{cccc}
1 & 0 & 0 & 0 \\
0 & 1 & 0 & 0 \\
0 & 0 & \cos \Delta & -\sin \Delta \\
0 & 0 & \sin \Delta & \cos \Delta
\end{array}\right)
$$

where $\Delta=-2 \tan ^{-1}\left(\frac{\cos \theta_{i} \sqrt{n^{2} \sin ^{2} \theta_{i}-1}}{n \sin ^{2} \theta_{i}}\right)$. Equations (2) and (3) govern the variations of polarization as a light ray interacts within a dielectric-filled material. 
The next challenge is: How can we design an optical component that is capable of guiding a ray to a special angular direction while simultaneously preserving its polarization? Technically, the total phase delay (or phase shift) during ray propagation must be kept as $2 \pi$, or an integral multiple of $2 \pi$, and the deflected angle between the exit and incident beams should match the designed value. The following section shows that a wedge plate is a possible candidate to achieve this goal.

\section{THE ISSUE OF POLARIZATION PRESERVATION AND ITS CONDITIONS}

In this section, we will show how to resolve the issue indicated in above section. We propose two types of light pipes, namely wedge and circular forms, the incident direction of light propagation could be rotated as necessary while still preserving beam polarization.

\subsection{The wedge plate form}

Part of the results was shown elsewhere [9], it is still included here for completeness. Much like the wedge plates considered in Refs. [12] and [13], Fig. 1(a) shows that a ray is incident to the narrow side of the wedge plate as long as the incident angle when the ray first strikes the top wedge surface is greater than the total internal reflection angle $\theta_{c}$. This ray can continuously propagate between the top and bottom surfaces of the wedge plate until it reaches the wide side (or the exit surface). To avoid unnecessary complexity, the wedge input and exit surfaces were cut such that their directions of surface normal are the same as the directions of incident and exit beams, respectively. In Fig. 1(a), $\alpha_{i}$ is the angle between the ray propagation direction and the horizontal axis after the $i^{\text {th }}$ total internal reflection, $\theta_{i}$ is the incident angle before the $i^{\text {th }}$ total internal reflection, $\theta_{v}$ is the wedge vertex angle, and $n$ is the refractive index of the plate material. Before leaving the wedge plate, this ray encounters $m$ times of total internal reflection.
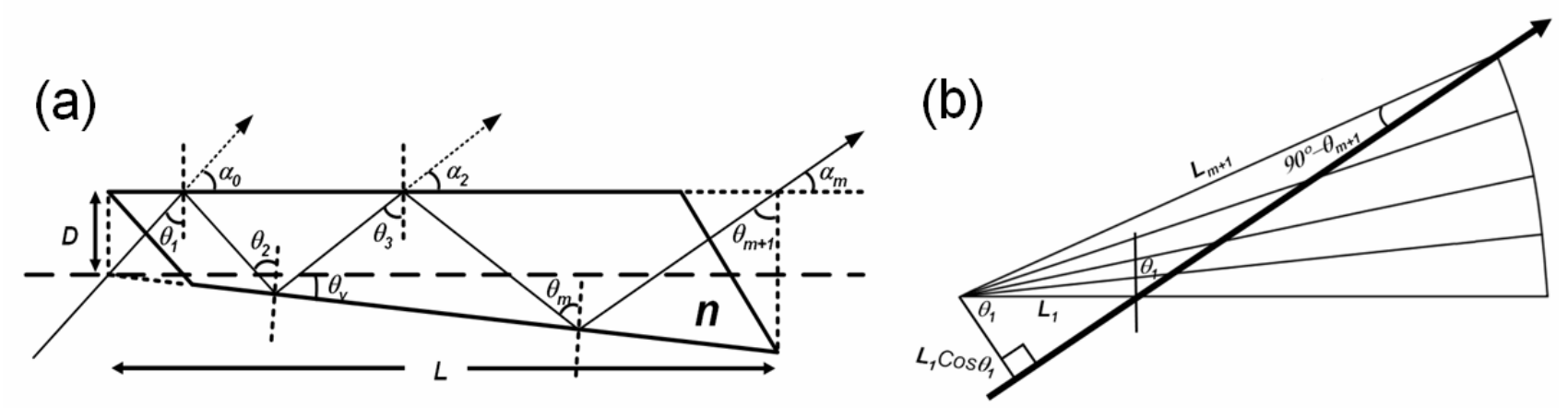

Figure 1: Schematic diagrams of ray propagating in (a) wedge plate, (b) a virtually folded wedge plate

Without loss of generality, we assume this ray finally escapes from the top surface of the wedge plate and $m$ is an even number. The geometry in Fig. 1(a) shows that $\theta_{i}=\theta_{1}+(i-1) \theta_{v}, \alpha_{i}=90-\theta_{i}-\theta_{v}$ (when $i$ is even), and the ray deflection angle $\Delta \alpha=\alpha_{0}-\alpha_{m}=m \theta_{v}$. In short, 


$$
\left\{\begin{array}{c}
\Delta \alpha=m \theta_{v} \\
\sum_{i=1}^{m}-2 \tan ^{-1}\left(\frac{\cos \left[\theta_{1}+(i-1) \theta_{v}\right] \sqrt{n^{2} \sin ^{2}\left[\theta_{1}+(i-1) \theta_{v}\right]-1}}{n \sin ^{2}\left[\theta_{1}+(i-1) \theta_{v}\right]}\right)=-2 \pi \cdot k
\end{array}\right.
$$

where $m$ is even and $k$ is an integer. After specifying the ray deflection angle $\Delta \alpha$, multiple solutions of $\left(\theta_{1}, \theta_{v}\right)$ can be derived from Eq. (4) with different values of $m$ and $k$. Equation (4) is the basic condition of a polarization-preserving angular shifter for a wedge plate. Formally, $\theta_{1}$ and $\theta_{v}$ are the dominate parameters in Eq. (4), but the real scale of the wedge plate, i.e., the width of input port $D$, and the total length of wedge $L$, provide the degree of freedom in an angular shifter.

(a)

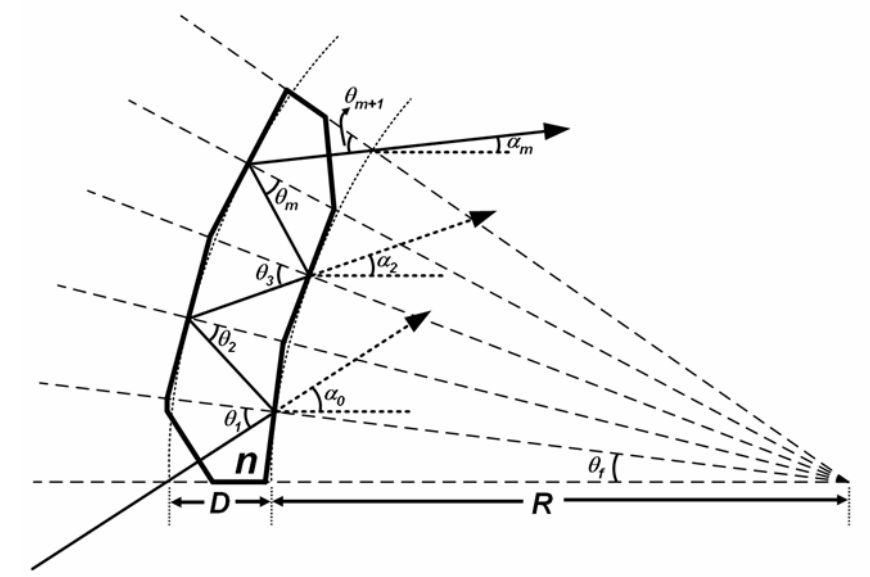

(b)

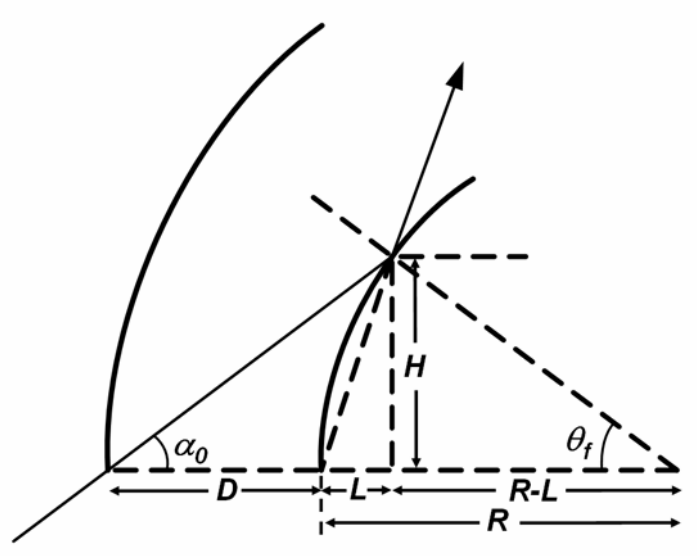

Figure 2: Schematic diagram of ray propagating in circular-bent light pipe: (a) multiple reflections; (b) first reflection.

\subsection{The circular-bent light pipe form}

It is worthwhile to demonstrate the approach to different form. One of the cases is to preserve polarization state of propagating ray is by take advantage of a circular-bent light pipe. Figure 2(a) shows a segment of the circular-bent light pipe, $\alpha_{i}$ is the angle between the ray propagation direction and the horizontal axis after the $i^{\text {th }}$ total internal reflection, $\theta_{i}$ is the incident angle before the $i^{\text {th }}$ total internal reflection, $\theta_{f}$ is the fan angle between $i^{\text {th }}$ and $(i+1)^{\text {th }}$ reflections, and $n$ is the refractive index of the plate material. To avoid complexity, we also cut the input and exit surfaces of circular-bent light pipe so that their directions of surface normal are the same as the directions of incident and exit beams. Similar to the deduction of wedge case in section 3.1, we have 


$$
\left\{\begin{array}{c}
\Delta \alpha=m \theta_{f} \\
\sum_{i=1(\text { odd })}^{m-1}-2 \tan ^{-1}\left(\frac{\cos \left(\alpha_{0}+\theta_{f}\right) \sqrt{n^{2} \sin ^{2}\left(\alpha_{0}+\theta_{f}\right)-1}}{n \sin ^{2}\left(\alpha_{0}+\theta_{f}\right)}\right)+\sum_{i=2(\text { even })}^{m}-2 \tan ^{-1}\left(\frac{\cos \alpha_{0} \sqrt{n^{2} \sin ^{2} \alpha_{0}-1}}{n \sin ^{2} \alpha_{0}}\right)=-2 \pi \cdot k
\end{array}\right.
$$

where $m$ is even and $k$ is an integer. After specifying the ray deflection angle $\Delta \alpha$, multiple solutions of $\left(\theta_{1}, \theta_{f}\right)$ can be derived from Eq. (5) with different values of $m$ and $k$, as the result of the wedge plate form . Equation (5) is the basic condition of a polarization-preserving angular shifter for a circular-bent light pipe. The width of input port $D$, and the radius of inner surface $R$, provides the degree of freedom in an angular shifter.

\section{NUMERICAL EXPLORATION}

For a numerical illustration for wedge plate type polarization-preserving angular shifter, consider a typical glass, BK7, where $n=1.51509$ at $632.8 \mathrm{~nm}$, and request $\Delta \alpha=30^{\circ}$. After solving, one solution was arbitrarily selected for demonstration: $m=10, \theta_{1}=49.46^{\circ}, \theta_{v}=3^{\circ}$, where the total phase delay is $\sim-2 \pi$, i.e., $-2 \pi-7.3 \times 10^{-4}$. Figure 1 (b) shows the series of a virtually folded wedge by which the multi-reflection ray path can be treated as a straight line. In Fig.1(b), $L_{l}$ is the length from wedge vertex to first total internal reflection position and $L_{m+l}$ is the length from wedge vertex to $(m+1)^{\text {th }}$ total internal reflection position. Simple mathematics prove that $L_{1}=D\left(\cot \theta_{v}+\tan \theta_{1}\right)$ and $L_{m+1}=\frac{L_{1} \cos \theta_{1}}{\sin \left(90^{\circ}-\theta_{m+1}\right)}$, and hence, the total length $L$ from the ray incident position to the exit position is

$$
\begin{aligned}
L & =L_{m+1}-L_{1}+D \tan \theta_{1} \\
& =D\left(\cot \theta_{v}+\tan \theta_{1}\right) \frac{\cos \theta_{1}}{\cos \left(\theta_{1}+m \theta v\right)}-D \cot \theta_{v}
\end{aligned}
$$

By Eq. (5), one can determine the exact size of the wedge plate by choosing the port width $D$ or the wedge length $L$.

In the numerical example above, $\theta_{v}=3.0^{\circ}$, and the thickness of the entrance port $D=5.0 \mathrm{~mm}$. By Eq.(6), the plate length $L$ is $264.5 \mathrm{~mm}$. For simplicity of presentation (without loss of generality), ignore the absorption for the time being. This study uses TracePro (version 4.1.5), a commercial simulation package, for numerical verification [14]. A collimated beam with $+45.0^{\circ}$ linearly polarization was propagated into the wedge plate at $\alpha_{0}=40.54^{\circ}$, and finally exited the wedge plate at $\alpha_{10}=10.54^{\circ}$ after 10 total internal reflections. Figure 3(a) shows the polarization states (the Stokes parameters) after the ray encounters even times of total internal reflection. Ellipse ratio $e$ of a linear polarized beam was considered and $e^{2}=I_{\min } / I_{\max }$ was calculated, where $I_{\max }$ and $I_{\min }$ are the maximum and minimum intensities of the optical beam, proportional to the squares of semimajor and semiminor axes of the ellipse, respectively, described by the beam's electric field vector. Results show that the output polarization beam has a good linear property, with the square of ellipse ratio $e^{2}=1.3332 \times 10^{-7}$ and the azimuth of polarization axis $44.51^{\circ}$. 
In the circular-bent light pipe case, we also consider a typical glass, BK7, where $n=1.51509$ at $632.8 \mathrm{~nm}$, but request $\Delta \alpha=60.0^{\circ}$. After solving, an arbitrarily solution was selected: $m=10, \theta_{1}=42.54^{\circ}, \theta_{f}=6.0^{\circ}$, where the total phase delay is $\sim-2 \pi$, i.e., $-2 \pi-1.497 \times 10^{-4}$. Figure $3(\mathrm{~b})$ shows the ray path when first reflection occurs inside the circular-bent light pipe, $D$ is the width of input port and $\mathrm{R}$ is the radius of light pipe inner surface, $H$ is the height of first total internal reflection point. By simple trigonometry, one can prove

$$
R=\frac{D \tan \alpha_{0}}{\sin \theta_{f}-\tan \alpha_{0}+\cos \theta_{f} \tan \alpha_{0}} .
$$

By Eq. (7), we can determine the exact size of the circular-bent light pipe by choosing the port width $D$ or the inner surface radius $R$. We choose the width $D=5.0 \mathrm{~mm}$ for numerical example then the inner surface radius $R$ is $46.11 \mathrm{~mm}$. The incident beam of numerical example was a collimated beam with right circular polarization, was propagated into the wedge plate at $\alpha_{0}=42.54^{\circ}$, and finally exited the wedge plate at $\alpha_{10}=-17.46^{\circ}$ after 10 total internal reflections. Results show that the output polarization beam has a good circular property, with the square of ellipse ratio $e^{2}=1-2.994 \times 10^{-4}$.

(a)

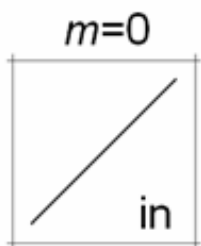

$S_{0}=1$

$S_{1}=0$

$S_{2}=1$

$S_{3}=0$

$e^{2}=0$

(b)

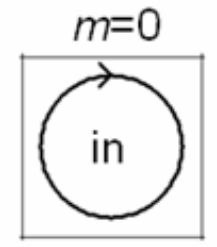

$S_{0}=1$

$S_{1}=0$

$S_{2}=0$

$S_{3}=1$

$e^{2}=1$

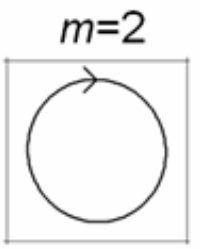

$S_{0}=0.9581$

$S_{1}=-1.1302 \times 10^{-16} S_{1}=2.9329 \times 10^{-16}$

$S_{2}=0.0348$

$S_{3}=0.9574$

$e^{2}=0.9299$

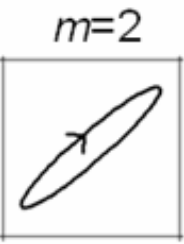

$S_{0}=0.9581$

$S_{1}=0$

$S_{2}=-0.9112$

$S_{3}=0.2961$

$e^{2}=0.0251$
$S_{2}=-0.9579$

$S_{3}=0.0119$

$\theta^{2}=3.8441 \times 10^{-5}$
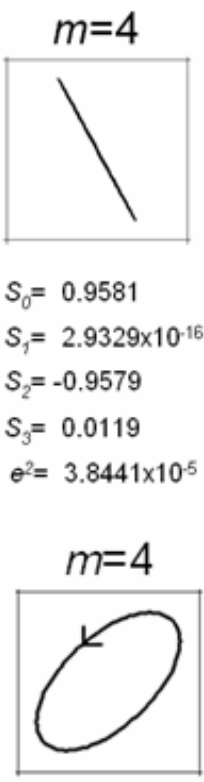

$S_{0}=0.9581$

$S_{1}=0$

$S_{2}=-0.5632$

$S_{3}=-0.7751$

$e^{2}=0.2596$

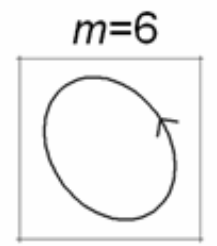

$S_{0}=0.9581$

$S_{1}=2.9866 \times 10^{-16}$

$S_{2}=-0.2501$

$S_{3}=-0.9249$

$e^{2}=0.5859$

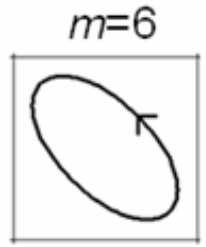

$S_{0}=0.9581$

$S_{1}=0$

$S_{2}=0.5631$

$S_{3}=-0.7751$

$e^{2}=0.2596$

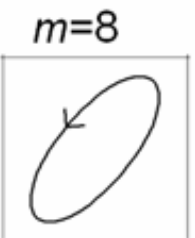

$S_{0}=0.9581$

$S_{1}=-1.2591 \times 10^{-16}$

$S_{2}=0.6854$

$S_{3}=-0.6694$

$e^{2}=0.1659$

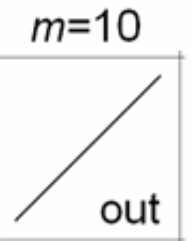

out

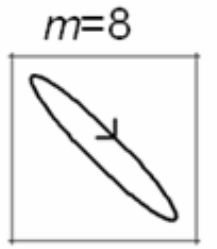

$S_{0}=0.9581$

$S_{1}=0$

$S_{2}=0.9112$

$S_{3}=0.2959$

$e^{2}=0.0251$

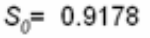

$S_{1}=-1.6861 \times 10^{-16}$

$S_{2}=0.9178$

$S_{3}=6.7029 \times 10^{-4}$

$e^{2}=1.3332 \times 10^{-7}$

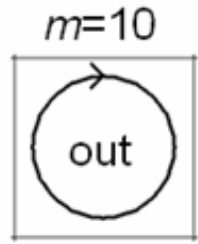

$S_{0}=0.9178$

$S_{1}=0$

$S_{2}=1.3742 \times 10^{-4}$

$S_{3}=0.9179$

$e^{2}=1-2.9941 \times 10^{-4}$

Figure 3: Polarization variations when ray propagated in (a) wedge plate; and (b) circular-bent light pipe.

\section{LIMITATION OF POLARIZATION-PRESERVING ANGULAR SHIFTER}


Physically, the limit of the ray deflection angle $\Delta \alpha$ of the wedge plate type polarization-preserving angular shifter should be less than the incident angle $\alpha_{0}$, while $\alpha_{0}$ should be less than $90.0^{\circ}-\theta_{c}$. For BK7 at a $632.8 \mathrm{~nm}$ wavelength, the critical angle $\theta_{c}$ is $41.3^{\circ}$, hence, the deflection angle $\Delta \alpha$ cannot be greater than $48.7^{\circ}$ when the wedge plate has an even number of reflections. Determining the limit of angular shifter is worthwhile, so this study explores the possible range of deflection angle $\Delta \alpha$ at specific vertex angles $\theta_{v}$, which range from $0.5^{\circ}$ to $5.0^{\circ}$ with a unit of $0.25^{\circ}$. Figure (4) shows the results, indicating that the available wedge vertex angle is limited from $0.0^{\circ}$ to $3.5^{\circ}$, although the range bounded up to $5^{\circ}$ has been checked, and 8 total internal reflections are required for the wedge plate to preserve the polarization after ray propagation.

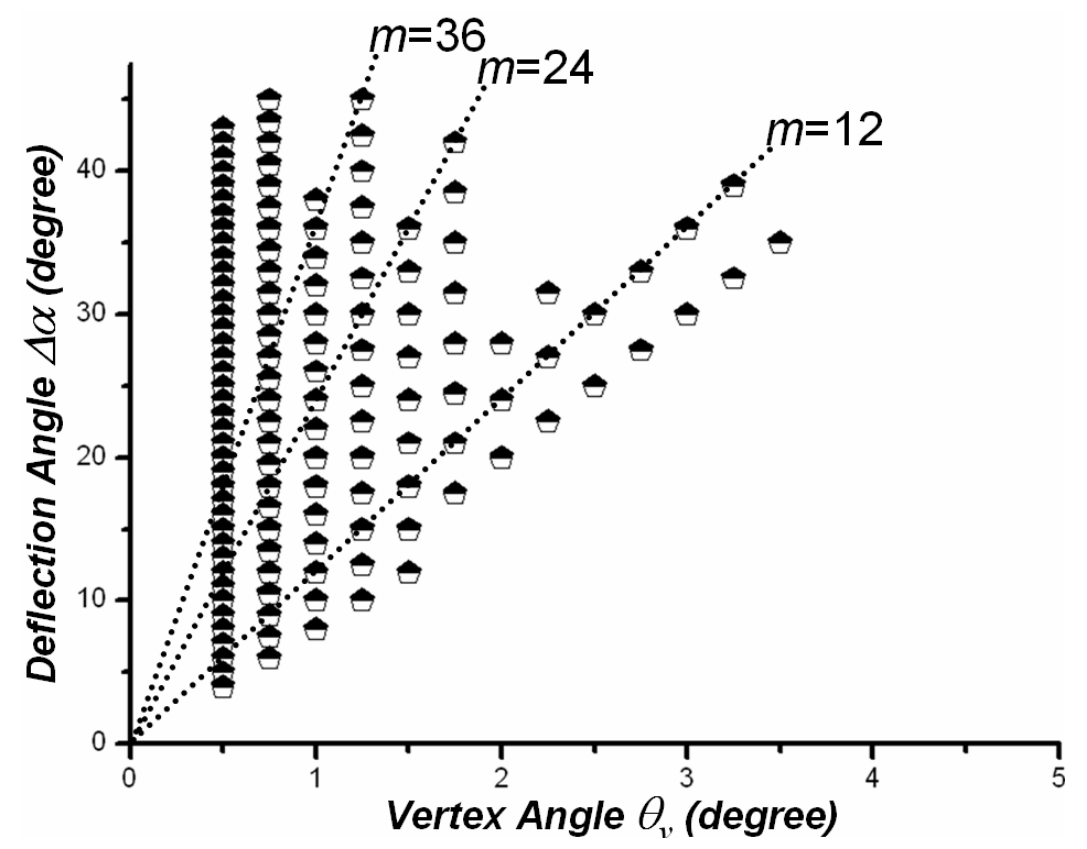

Figure 4: Possible ray deflection angles versus wedge vertex angle. The vertex angles $\theta_{v}$ is picked up from $0.5^{\circ}$ to $5.0^{\circ}$ with a unit of $0.25^{\circ}$ to determine the possible ray deflection angle.

A greater number of reflections increases the system dimensions and enlarges the phase difference caused by incident angle error. Figure (4) shows different solutions for three reflection numbers $m=12,24$, and 36 highlighted by dashed lines. This figure indicates that the maximal deflection angle can reach $39.0^{\circ}$ when the deflection number is 12 , while twice as many reflections increase the deflection angle to $42.0^{\circ}$, and three times as many reflections contributes an additional 3 degrees of deflection angle range.

For simplicity, this study only shows an even number of reflections for both cases. Nevertheless, one can simply multiply the deflection angle $\Delta \alpha$ twice by means of the wedge plate case with an odd number of total internal reflections. On the other hand, the circular-bent light pipe has an advantage than the wedge plate on the unlimited deflection angle range. Furthermore, utilizing multiple polarization-preserving wedges can also increase the deflection angle. 


\section{TOLERANCE ISSUE}

A polarization-preserving wedge or bent light pipe can be manufactured using glass molding or plastic injection. The accuracy of the incident plane angle and incident beam angle plays an important role in preserving the polarization state. Figures 1(a) and 2(a) show that the polarized beam normally incidents to the obliquely-cut input surfaces and exits from the output surfaces after $m$ total reflections in both cases. Incident angle errors negatively affect polarization-preserving shifter performance because additional phase delay arises with these errors occur.

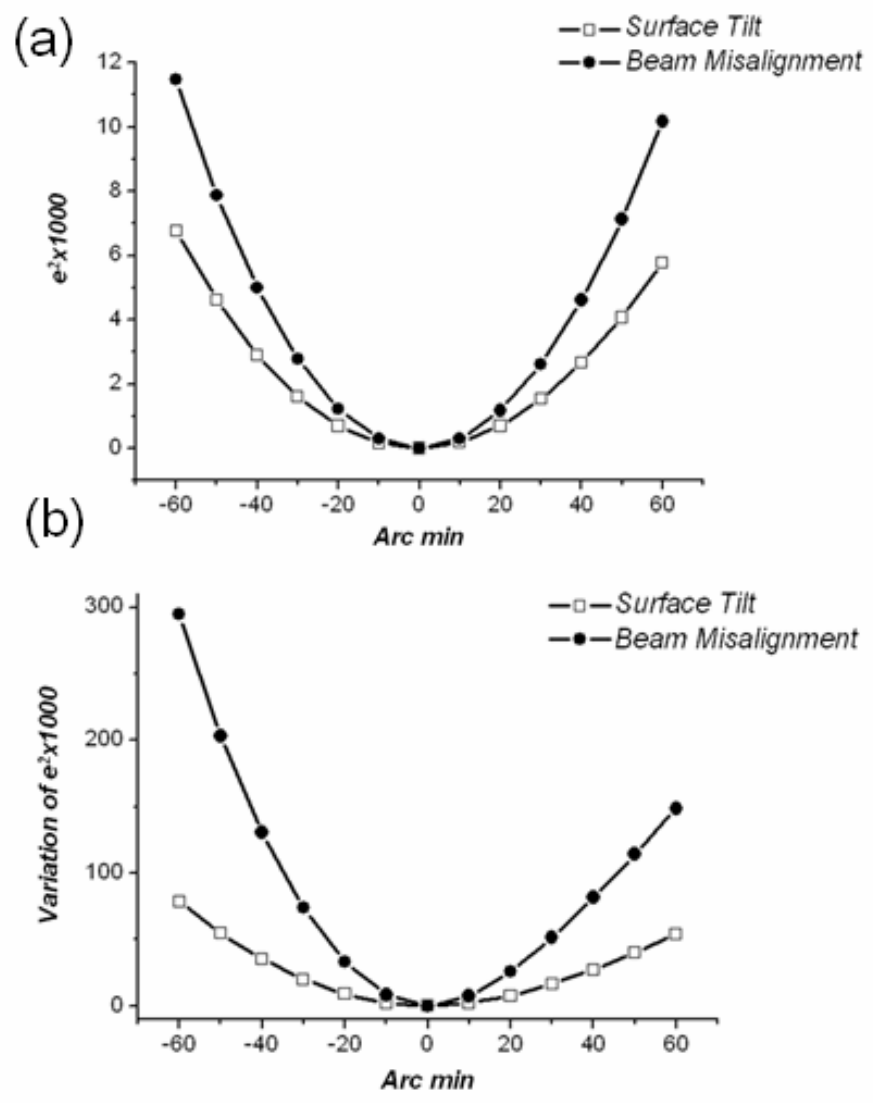

Figure 5: Performance degradation caused by incident surface tile and beam misalignment for:

(a) wedge plate; (b) circular-bent light pipe.

This paper discusses two variations of ray propagation angles. For the wedge plate case, first, if the ray normally incident to the input surface which is tilted by a small angle $\delta_{s}$, i.e. the incident angle $\alpha_{0}{ }^{\prime}=\alpha_{0}+\delta_{s}$, then the ray angle after the $m^{\text {th }}$ reflection for wedge plate is $\alpha_{m}{ }^{\prime}=\alpha_{0}+\delta_{s}+m \theta_{v}$ and the ray exit angle becomes $\alpha_{\text {out }}=\alpha_{0}+\sin ^{-1}(n \sin \delta)+m \theta_{v}=\alpha_{m}+\sin ^{-1}\left(n \sin \delta_{s}\right)$. The deflection angle of the output ray can be altered by another small angle $\sin ^{-1}\left(n \sin \delta_{s}\right)-\delta_{s}$, i.e., $\Delta \alpha^{\prime}=\Delta \alpha+\sin ^{-1}\left(n \sin \delta_{s}\right)-\delta_{s}=m \theta_{v}+\sin ^{-1}\left(n \sin \delta_{s}\right)-\delta_{s}$. Secondly, when each surfaces of the polarization-preserving wedge is manufactured identically, but the misalignment of 
the incident beam with the incident plane decreases performance. When the incident beam is tilted a by small angle $\delta_{i}$, i.e. the incident angle $\alpha_{i n}=\alpha_{0}+\delta_{i}$, the ray angle before first reflection should be adjusted to $\alpha_{0}{ }^{\prime}=\alpha_{0}+\sin ^{-1}\left(\frac{\sin \delta_{i}}{n}\right)$. The ray angle after the $m^{\text {th }}$ reflection is then $\alpha_{m}{ }^{\prime}=\alpha_{0}+\sin ^{-1}\left(\frac{\sin \delta_{i}}{n}\right)+m \theta_{v}$, and the ray exit angle finally becomes $\alpha_{\text {out }}=\alpha_{0}+\delta_{i}+m \theta_{v}=\alpha_{m}+\delta_{i}$. Thus, an angular error in the output beam is the same as the incident beam's angular error. In other words, the ray deflection angle $\Delta \alpha$ can remain unchanged when the incident beam is misaligned.

For the circular-bent light pipe case, the angular errors of output beam direction due to surface tile or beam misalignment have the same form as that in the wedge plate case. One can easily determine the angular shift of output beam by replace the wedge vertex angle $\theta_{v}$ as the fan angle $\theta_{f}$.

Angular errors on incident surfaces or incident beam alignment do not seriously affect the deflection angle (or output ray direction) in both scenarios above, but variation in the output beam's polarization state is unavoidable. The phase shift contributed by input and output surfaces, as deduced in Eq. (2), must be considered as well. Figures 5(a) and 5(b) investigate variations of the square of ellipse ratio $e^{2}$ for both the wedge plate and the circular-bent light pipe cases in section 4, where angular errors occur in the input surface, and incident beam alignment. The simulation results in Figs. 5(a) and (b) show that variations of $e^{2}$ caused by one degree of the input surface error are less than $1.0 \%$ (for wedge plate) and 30.0\% (for circular-bent light pipe). The results also show the impact of one degree of misalignment in the incident beam. The impact of the incident beam misalignment is similar to that of input surface tilt.

\section{CONCLUSIONS}

In conclusion, this study proposes and numerically demonstrates that a wedge plate or a circular-bent light pipe can angularly shift a collimated beam while maintaining its polarization state. This study provides the conditions for polarization preservation and the method of estimating the system scale to allow others to design and manufacture a polarization-preserving angular shifter based on this approach. For simulation investigation, two numerical cases of different types of polarization-preserving shifters were discussed. Simulation results show that this method can indeed transfer a polarization beam into a desired angle while maintaining its polarization state. Tolerance analysis shows that the incident beam angle's angular error does not affect the deflection angle and the variation of squared ellipse ratio can be kept under $1.0 \%$ (for wedge plate) and $30.0 \%$ (for circular-bent light pipe) with 1 degree accuracy for the incident beam angle.

Polarized beams are widely used in measuring thin film, biological tissue, glass surface stress, and so forth. This study helps designers understand ray polarization behavior within dielectric-fill material and control the phase difference by properly choosing wedge or light pipe parameters. This method can easily be extended to other applications of polarization controlling mechanisms. For example, it is possible to replace the requirement of $2 \pi$ total phase with another value to create a defecting phase retarder. 
Acknowledgement: This work was supported by the National Science Council of Taiwan, R.O.C., under contract no. NSC 96-2628-E-009-019-MY3. We thank the Lambda Research Corp. for the educational support of the simulation package, TracePro.

\section{REFERENCES}

[1] M. L. Faupel, S. B. Bambot, T. Harrell, and A. Agrawal, "Multi-modal optical tissue diagnostic system,” U.S. Patent \#6975899, (December 13, 2005).

[2] R. M. Azzam and N. M. Bashara, Ellipsometry and polarized light, (North-Holland, New York, 1989).

[3] R. M. Azzam, "Displacement of a monochromatic light beam parallel to itself without change of polarization," Opt. Lett., 7, 80-82 (1982).

[4] R. M. Azzam and M. Emdadur Rahman Khan, "Polarization-preserving single-layer-coated beam displacers and axicons,” Appl. Opt., 21, 3314-3322 (1982).

[5] E. Cojocaru, "Polarization-preserving totally reflecting prisms," Appl. Opt., 31, 4340-4342 (1992).

[6] Z. P. Wang, W. M. Sun, S. L. Ruan, C. Kang, Z. J. Huang, and S. Q. Zhang, "Polarization-preserving totally reflecting prisms with a single medium layer," Appl. Opt., 36, 2802-2806 (1997).

[7] E. J. Galvez, “Achromatic polarization-preserving beam displacer,” Opt. Lett., 26, 971-973 (2001).

[8] A-C Hsu, C.-F. Ho, and J.-L. Chern, "Tilting tolerance analysis of a broadband polarization-preserving beam displacer", Appl. Opt., 41, 5956-5962 (2002).

[9] Part of the results on wedge-plate form has been published in Y.-K. Cheng and J.-L. Chern, "Polarization-preserving angular shifter,” J. Opt. Soc. Am. A 25, 1558-1563 (2008). The paper was accepted after this submission to SPIE presentation.

[10] M. Born and E. Wolf, Principles of Optics, 7th ed. (Cambridge, University, New York, 1999), pp. 42-43 and 50-51.

${ }^{111]}$ E. Collett, Polarized light: fundamentals and applications, (Marcel Dekker. Inc., New York, 1992), pp. 139-161.

[12] Y.-K. Cheng, S.-N. Chung, and J.-L. Chern, "Analysis and reduction of dark zone in ultra-thin wedge plate display", J. Soc. Inf. Disp. 14, 813-818 (2006).

[13] Y.-K. Cheng, S.-N. Chung, and J.-L. Chern, “Aberration analysis of a wedge-plate display system”, J. Opt. Soc. Am. A, 24, 2357-2362 (2007).

[14] see http://lambdares.com for technical information 